\title{
Claustrophobia Game: Design and Development of a New Virtual Reality Game for Treatment of Claustrophobia
}

\begin{abstract}
Background: Claustrophobia or fear of closed spaces is the most common of phobias that is typically categorized as an anxiety disorder. Different methods have been proposed for treatment of phobias that one of the most recent and successful of these methods is applying virtual reality (VR) technology and simulating computer-generated environment. In this regard, the purpose of this research is design and development of a software game called "Claustrophobia Game" for treatment of claustrophobia using VR. Methods: In the Claustrophobia Game project, two closed spaces, including an elevator and a magnetic resonance imaging (MRI) device, were designed and implemented in the form of a VR computer game. To design this game, environments and scenario of the game were prepared in collaboration with a psychiatrist expert. Implementation of the software game was developed in the unity three-dimensional (3D) game engine and the programming of it was done by the $\mathrm{C} \#$ language. In addition, a personal computer and the Oculus Rift VR glasses were utilized for running and testing the Claustrophobia Game. Results: To evaluate, we tested the game by 33 participants (14 men, 19 women, average age 24.6 years). In this regard, the Claustrophobia Game was considered from two aspects: psychology and playability using two questionnaires. Statistical analysis of the obtained data by the Excel software showed that all playability factors were "good" performance. In addition, the mean of obvious anxiety was decreased after playing the game. Conclusion: The promising results demonstrate that the game has an appropriate performance and can help to treat the Claustrophobia.
\end{abstract}

Keywords: Claustrophobia, game software development, phobia, treatment game, virtual reality

\section{Introduction}

Phobia is a type of psychiatric disorders that are classified in the group of anxiety disorders. In this disorder, the patient suffers from extreme fear when faced with a special object or situation. Statistically, phobia is the most common anxiety disorder that approximately $15 \%$ of women and $7 \%-8 \%$ of men have this disease. Among types of phobias, Claustrophobia is more common than other phobias. This phobia is a kind of unreasonable fear that occurs in enclosed spaces such as elevator, tunnel, computed tomography, and magnetic resonance imaging (MRI) devices. ${ }^{[1]}$

Different methods have been presented for the treatment of phobias that the most commonly used method is exposure therapy. ${ }^{[2]}$ In this method, the patient is gradually faced with his/her anxiety situations to realize his/her irrational

This is an open access journal, and articles are distributed under the terms of the Creative Commons Attribution-NonCommercial-ShareAlike 4.0 License, which allows others to remix, tweak, and build upon the work non-commercially, as long as appropriate credit is given and the new creations are licensed under the identical terms.

For reprints contact: reprints@medknow.com fear; however, it is a costly and time-consuming process. On the other hand, nowadays, new technologies such as virtual reality (VR) widely used in medicine for preventive care, diagnosis, treatment, and learning/training of clinical skills. $^{[3]}$ VR defines as a technology that creates simulated environments to mimic real-world situations. ${ }^{[4]}$ This technology allows users to interact with a computer-generated environment that simulates the physical presence of a person in the real or imagined world ${ }^{[5,6]}$ Applying advanced input/output devices and graphical software tools, users feel that they are in a world similar to the real world and can interact with the environment, objects, and people. ${ }^{[7]}$ Since the use of VR can turn threatening and tedious conditions into safe and enjoyable states; in recent years, employing this technology has been considered for the treatment of many mental illnesses

\footnotetext{
How to cite this article: Rahani VK, Vard A, Najafi M. Claustrophobia Game: Design and development of a new virtual reality game for treatment of Claustrophobia. J Med Sign Sens 2018;XX:XX-XX.

Received: June, 2018. Accepted: August, 2018.
}

\section{Vida Kabiri Rahani ${ }^{1}$, Alireza Vard², Mostafa Najafi ${ }^{3}$}

${ }^{1}$ Department of Information Technology, Safahan Institute of Higher Education, ${ }^{2}$ Department of Biomedical Engineering, School of Advanced Technologies in Medicine and Medical Image and Signal Processing Research Center, Isfahan University of Medical Sciences, ${ }^{3}$ Department of Psychiatry, School of Medicine, Isfahan University of Medical Sciences, Isfahan, Iran 
especially for phobias. ${ }^{[8]}$ In the last years, various studies have been carried out in this field that the results of them show that applying VR can shorten the period of treatment and save time and cost. Furthermore, it can help psychologists to have better control on patients and the treatment process. On the other hand, In spite of the feeling of being in a real environment, patients can easily overcome to their fear due to sense of presence in the safe and attractive environment. ${ }^{[9-13]}$

Some of the reported studies about the treatment of Claustrophobia using VR technology are shown in Table 1. In each of them, different game environments have been designed and evaluated that the positive results of these studies can verify the success of using VR technology in the treatment process of Claustrophobia.

Regarding the importance of Claustrophobia among of anxiety disorders and promising results of previous work, utilizing VR environments in the psychiatric centers of universities, hospitals, and clinics can be very useful and helpful for research and treatment purposes. Accordingly, in this paper, we present a software game called "Claustrophobia Game" that has been developed to deal with Claustrophobia using VR technology and explain the various stages of design, implementation, and evaluation of this software. The main contribution of this project is as follows:

1 Respect to localization of the software game. This software has been adapted to the linguistic, cultural, and technical requirements of our country

2 To involve MRI machine as a part of claustrophobia game. Design and develop of the MRI part for Claustrophobia have been done for the first time in this project.

\section{Materials and Methods}

\section{Game development process}

According to the block diagram of Figure 1, design and development of the Claustrophobia Game were done in four stages including pre-production, game design, game implementation, and game evaluation. Each part of this block diagram is described in the following of this section.

- Stage 1: Preproduction - In the first stage, previous research and methods of game development in this field were studied. In addition, hardware and software tools applied in similar products were considered. Then, the appropriate hardware platform and software tools were selected and provided for this project

- Stage 2: Game design - In the second step, together with a psychiatrist expert, the game environments were designed, and the game scenario including levels, goals, challenges, and rules were prepared according to Table 2

- Stage 3: Game implementation - In this stage, game environments and objects inside them were created by graphical software tools and game engines. In addition, the game dynamics consisting of visual effects, sound effects, and movements of objects were programmed in the programming software frameworks

- Stage 4: Game evaluation - In the final stage, the produced game software was tested and evaluated by

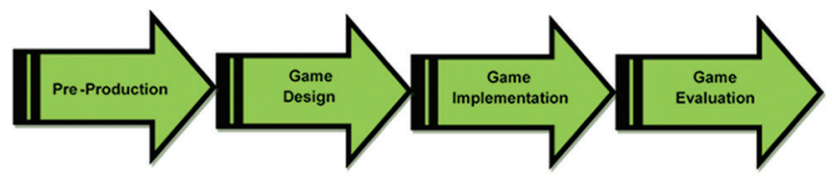

Figure 1: Stages of the game development process

\begin{tabular}{|c|c|}
\hline Reference & Description \\
\hline Botella et al. $1999^{[14]}$ & $\begin{array}{l}\text { In this research, a VR elevator was used for the treatment of claustrophobia. In this regard, an 8-session } \\
\text { treatment consisting of VR exposure therapy was designed. This procedure used to treatment of one patient } \\
\text { and results indicated the effectiveness of the VR procedure for the treatment of claustrophobia }\end{array}$ \\
\hline Botella et al. $2000^{[15]}$ & $\begin{array}{l}\text { A 8-level VR game including an elevator and a building was tested in this study. In every level, the game } \\
\text { would be harder. The game was evaluated on four patients and the reported results were promising }\end{array}$ \\
\hline Malbos et al. $2008^{[11]}$ & $\begin{array}{l}\text { A software VR game with houses, corridors, caves, channels, basement and elevator was considered. In an } \\
8 \text {-session treatment, six patients used it and results pointed out a remarkable reduction in fear of the enclosed } \\
\text { space and improvement of life quality }\end{array}$ \\
\hline Bruce and & Four VR rooms that gradually become darker and smaller were designed in this project. A Non-clinical \\
\hline Regenbrecht $2009^{[10]}$ & $\begin{array}{l}\text { laboratory experiment with participating of eighteen volunteers was performed in this work. They reported that } \\
\text { anxiety decreased on the successive use of their system }\end{array}$ \\
\hline Bouchard et al. 2014[3] & $\begin{array}{l}\text { In this work, different VR environments comprising of elevator, cave, tunnel and maze were designed. These } \\
\text { VR environments were employed for treatment of two patients. The reported results demonstrated positive } \\
\text { effects of VR in treatment of claustrophobia }\end{array}$ \\
\hline $\begin{array}{l}\text { Christofi and Michael- } \\
\text { Grigoriou 2016 }\end{array}$ & $\begin{array}{l}\text { In this study, several virtual environments including building with different rooms, tunnel and cellar, } \\
\text { which they differ in a number of characteristics, were investigated. Eighteen students participated in a pilot } \\
\text { experimental study. Their results show that status and characteristics of environments can effect on increasing/ } \\
\text { decreasing of people anxious }\end{array}$ \\
\hline
\end{tabular}

VR - Virtual reality 
some volunteer participants to determine playability and effectiveness of it.

\section{Software tools}

Nowadays, to develop video games, game engine toolkits are used. The game engine is a software framework that provides a set of core components for development of computer games including rendering tools for the creation of $2 \mathrm{D}$ or $3 \mathrm{D}$ graphical models, animation and physics tools, audio system, scripting, and so on. In this project, we utilized Unity3D (https://unity3d.com/) as the game engine to create the 3D virtual environments, standard 3D models of objects, and sound effects of the game. In addition, for creation of some complex models, we employed other computer graphics tools consisting of 3D Studio MAX (http://autodesk.com/3dsmax, Maya (http:// www.autodesk.com/maya), Adobe Photoshop (http://adobe. com/photoshop) and imported these models to Unity3D framework. To provide game dynamics, such as elevator movements, play movies and sounds, player movements, and camera settings, we utilized Microsoft Visual Studio C\# (https://visualstudio.com/) as a programming platform and added code files to the Unity3D as script files.

\section{Hardware system}

In this project, we selected the personal computer (PC) platform for running the game software and a head-mounted display (HMD) glasses to create sense of visual immersion in the participants. Technical specifications of the computer system and the HMD glass employed for testing in this project are as follows:

- Computer system: A desktop PC with a central processing unit core i7 4.2 GHz, Graphic Card Asus DUAL-GTX1070-O8G, RAM 16 G DDR4, and the Windows 10 operating system

- HMD glasses: The Oculus Rift glasses which release in 2016 (https://www.oculus.com/en-us/rift/).

\section{Game description}

In this section, different parts of the Claustrophobia Game are briefly explained. When the game software is run, the game is started with "welcome page" and

\begin{tabular}{llll}
\hline \multicolumn{3}{c}{ Table 2: Components of the game scenario } \\
\hline Level & Goal & Challenge & Rule \\
\hline Elevator & $\begin{array}{l}\text { Treat the } \\
\text { fear of } \\
\text { elevator }\end{array}$ & $\begin{array}{l}\text { Stay in closed } \\
\text { space of the } \\
\text { elevator and } \\
\text { move up to the } \\
10^{\text {th }} \text { floor }\end{array}$ & $\begin{array}{l}\text { To reach the } 10^{\text {th }} \text { floor, } \\
\text { player must move up } \\
\text { from the first floor to the } \\
10^{\text {th }} \text { floor step-by-step } \\
\text { without fear or anxiety }\end{array}$ \\
MRI & $\begin{array}{l}\text { Treat } \\
\text { Ability to watch }\end{array}$ & $\begin{array}{l}\text { If the player can't finish } \\
\text { machine fear } \\
\text { a video clip when } \\
\text { the whole MRI video clip } \\
\text { the patient is } \\
\text { machine } \\
\text { placed inside of } \\
\text { the MR scanner }\end{array}$ & $\begin{array}{l}\text { due to fear or anxiety, he/ } \\
\text { she must start playing it } \\
\text { from the beginning }\end{array}$ \\
\hline
\end{tabular}

MRI - Magnetic resonance imaging enters to "login page" [Figure 2a]. If the user has already registered, he/she can log in by entering his/her full name. When the user has not registered, he/she will be transferred to a "registration page" [Figure 2b]. In this page, some information such as name, age, gender, heart rate, blood pressure, and breath count is taken from the user and saved in the database. After registering and logging in, the user enters the main menu screen with three options [Figure 2c]:

- The first option is displayed a video clip, created by Ulead VideoStudio Software (https://www. videostudiopro.com/) that introduces the fear of closed environment [Figure 2d]

- The second option consists of an elevator in a ten-story building that design for VR exposure therapy in patients with fear of closed spaces [Figure 2e]. The building includes interesting outdoor and indoor virtual environments that the therapist can step-by-step exposes patients with their fear in some controlled situations. The internal lobby of building [Figure 2f] including directional signs that led the user to an elevator or a relaxation room [Figure 2g]. When the user goes to the relaxation room, a video clip containing some relaxation pictures and human voices with music background starts to play that help the user to relax himself/herself [Figure 2h]. On the other hand, when the user moves to the elevator, he/she faces to sometime panels on the wall that user can determine the duration of presence inside the elevator by selecting each of these buttons [Figure 3a]. After choosing a time panel, the user can go to inside the elevator [Figure 3b] and by pressing buttons of it, the door of elevator is closed and move to the upper floors. According to the selected time, user stays inside the closed space of elevator, but each time the player suffers from a panic attack, he/she can leave the elevator in each floor and go to the relaxation room

- The third option in the Claustrophobia Game includes a building with an MRI room. When the user selects this section, he/she enters in the VR building and by following of directional green signs; the player can reach the MRI room [Figure 3c]. There is a MRI machine inside that room which the player can interact with it. When user gets really close to the MRI machine and ready to place inside of the MR scanner, a video clip plays that shows inside of the MRI machine [Figure 3d].

Furthermore, in different parts of the game, sound effects such as sound of open/close elevator's door or sounds of birds and water and a guiding voice that directs the players in the game as well as attractive design of environments encourage users to continue the game. However, in any part of the game, when the user wants to exit, he/she can save his/her status and information and then exits. 
Performance evaluation

\section{Participants}

In this research, there were two groups of participants. The first group consists of 14 patients who are afraid of closed environments. The second group consists of 19 volunteers

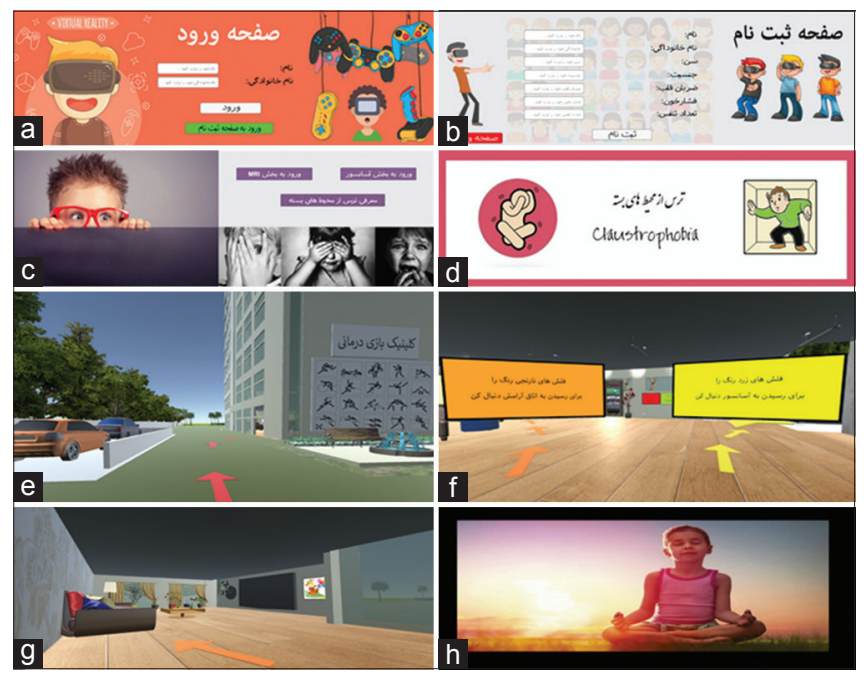

Figure 2: Screenshots of the of the Claustrophobia Game part 1: (a) Login page, (b) registration page, (c) main menu, (d) play a video clip to introduce the fear of closed environment, (e) outdoor view of the building that has the elevator, $(f)$ indoor view of the building that has the elevator, $(g)$ relaxation room, (h) display a relaxing video clip in the relaxation room

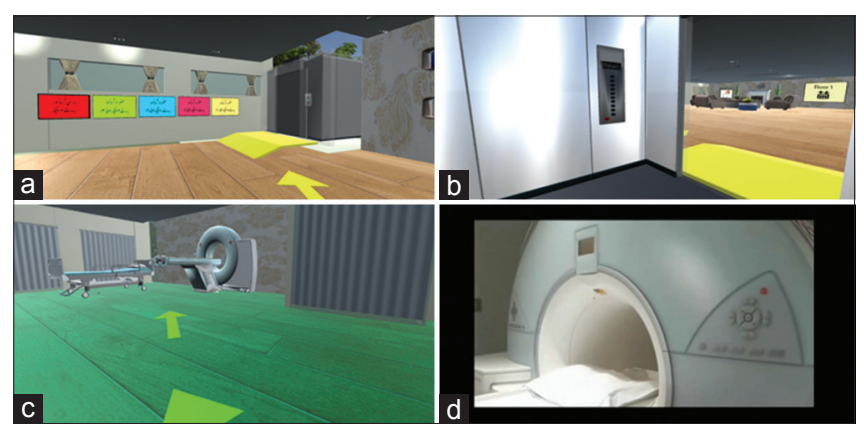

Figure 3: Screenshots of the of the Claustrophobia Game part 2: (a) Exterior view of the elevator and selecting time panels, (b) interior view of the elevator, (c) magnetic resonance imaging room, (d) play a video clip that shows inside of the magnetic resonance imaging machine

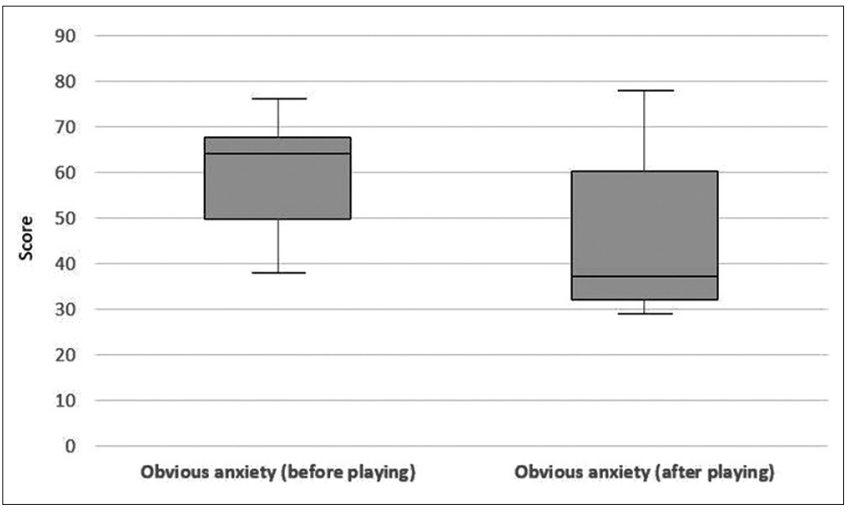

Figure 4: Boxplot of obtained scores from psychology questionnaires who do not have trouble with closed environments. Among of these 33 people, there were 14 men and 19 women with average age of 24.6 years (range 15-36 years).

\section{Data collection and analysis}

We evaluated the ClaustrophobiaGame from two aspects: psychology and playability. First, to measure the effectiveness of this game for the treatment of claustrophobia, we used Spielberger questionnaire, ${ }^{[17]}$ which reliability and validity of it have been accepted in this field. The psychology questionnaire contains 20 items that measure obvious anxiety. Each item in this questionnaire is scored from 20 to 80 . According to the principle stated in the paper, ${ }^{[17]}$ the score for each item is calculated and based on Table 3, the stress level of a person is determined.

Second, to measure playability of the game, we extracted seven important factors from previous articles ${ }^{[18-23]}$ and designed a playability questionnaire by considering the opinions of the experts in the video game field. Factors and definition of them, utilized in the playability questionnaire, have been presented in Table 4 and each factor is scored from 1 to 7 .

Then, the average percentage of the scores for each factor is calculated. In this regard, the answers from all participants are gathered. Next, the average score is calculated for each factor, and the percentage of it is computed. Then, according to Table 5, the performance for each factor is determined.

The psychology questionnaire was only given to patients, before and after playing the game, but the playability questionnaire was given to all the players (patients and volunteers) after playing the game. Both questionnaires were analyzed with statistical methods in the Excel software.

\section{Results and Discussion}

As mentioned in the previous section, we considered the game from two aspects. In aspect of playability, the obtained results have been shown in Table 6. In this table, five statistical parameters including minimum, maximum, mean, standard deviation (SD), and mean percentage are indicated. These parameters were obtained from 33 playability questionnaires and calculated for seven factors of Table 4.

\begin{tabular}{|c|c|c|}
\hline Stress type & Scores & Stress level \\
\hline Obvious & From 20 to 31 & Mild anxiety \\
\hline \multirow[t]{5}{*}{ anxiety } & From 32 to 42 & Medium to low anxiety \\
\hline & From 43 to 53 & Moderate to high anxiety \\
\hline & From 54 to 64 & Relatively severe anxiety \\
\hline & From 65 to 75 & Severe anxiety \\
\hline & From 76 to up & Very intense anxiety \\
\hline
\end{tabular}


Table 4: Factors and definition of them used in the playability questionnaire

\begin{tabular}{ll}
\hline Factor & Definition \\
$\begin{array}{l}\text { Emotion } \\
\text { Motivation }\end{array}$ & $\begin{array}{l}\text { Emotional response of the user to game's triggers, interactions, feeling of change in behavior, player's sense of safety } \\
\text { Fatisfaction }\end{array}$ \\
$\begin{array}{l}\text { Features of the game that excite players to realize actions of the game and encourage them to continue the game to end } \\
\text { Degree of user pleasure in using the game, which includes attractiveness and flexibility of the game, creation of self- } \\
\text { Immersion } \\
\text { Sense in which a player becomes attracted and involved in the game. More challenging, more exciting and more cultural } \\
\text { proximity can more immerse the player in the game }\end{array}$ \\
Esfectiveness \\
$\begin{array}{l}\text { Degree of fitness and compatibility between current activity and the goal. Good game structure, reduction of attempts to } \\
\text { overcome the challenges, appropriate end regarding to expectation of each user and increase precision can enhance effectiveness } \\
\text { Indicator to measure the ease of use of the game. More easily use of the game, and no inconsistency in the game can increase } \\
\text { usability }\end{array}$
\end{tabular}

In Table 6 , the mean percentages of all playability factors are more than $60 \%$ and $<80 \%$ that according to Table 5 , all of them have "good" performance.

Boxplot and statistical results calculated from psychology questionnaires are as shown in Figure 4 and Table 7, respectively. In Table 7, four statistical metrics consisting of minimum, maximum, mean, and SD computed for obvious anxiety before and after playing the game.

As seen the scores in boxplot and the means in Table 7, the obvious anxiety after playing the game was less than before playing the game. It shows that the game can help users to decrease their fear from the closed spaces. To determine statistically significant differences in the means, we performed a paired $t$-test between means of obvious anxiety of 14 people before and after playing the game (at a 0.05 level of significance). In this test, we assumed that the null hypothesis is: "There is no significant difference between the mean of obvious anxiety before and after playing the game." The obtained $P=0.013$ and $<0.05$, so that the null hypothesis is rejected. As a result, this test indicates a significant difference between the mean of obvious anxiety before and after playing the game.

\section{Conclusion}

In this paper, we proposed a VR software game for the treatment of Claustrophobia. At first, we explained the four-stage game development process including preproduction, game design, game implementation, and game evaluation and stated the specification of software tools and hardware systems that utilized for developing and testing the game. Next, we briefly described different parts of the game consisting of environments, graphical user interfaces, and how to interact with the game. Then, description of participants; types of used questionnaires to determine playability, and psychology performance of the game were mentioned in the performance evaluation subsection. Finally, we reported that the results obtained from statistical analysis of the questionnaire data. The results showed that all playability factors had "good"
Table 5: Performance of playability factors based on the average percentage of the obtained scores

\begin{tabular}{ll}
\hline Average scores $(\mathbf{\%})$ & Performance \\
\hline$<60$ & Bad \\
Between 60 and 80 & Good \\
$>80$ & Very good \\
\hline
\end{tabular}

Table 6: Statistical analysis results of playability questionnaire

\begin{tabular}{lccccc}
\hline Factor & Minimum & Maximum & Mean & SD & $\begin{array}{c}\text { Mean } \\
\text { percentage }\end{array}$ \\
\hline Emotion & 4 & 6.5 & 5.41 & 0.71 & 77.3 \\
Motivation & 3.5 & 7 & 5.58 & 0.95 & 79.7 \\
Satisfaction & 4.5 & 6.5 & 5.51 & 0.52 & 78.6 \\
Immersion & 4.6 & 6.8 & 5.60 & 0.62 & 80 \\
Effectiveness & 4.3 & 6.8 & 5.53 & 0.77 & 78.9 \\
Usability & 3.5 & 6.5 & 5.39 & 0.71 & 76.9 \\
Learnability & 2.4 & 6.8 & 5.42 & 0.99 & 77.5 \\
\hline
\end{tabular}

Table 7: Statistical analysis results of psychology questionnaire

\begin{tabular}{lcccc}
\hline Variable & Minimum & Maximum & Mean & SD \\
\hline $\begin{array}{l}\text { Obvious anxiety } \\
\text { (before playing) }\end{array}$ & 38 & 76 & 59.57 & 11.31 \\
$\begin{array}{l}\text { Obvious anxiety } \\
\text { (after playing) }\end{array}$ & 29 & 78 & 45.07 & 17.01 \\
\hline
\end{tabular}

SD - Standard deviation

performance and obvious anxiety could decrease after playing the game.

As a further study, we are going to assess the efficiency and usability of the proposed game in medical clinics as a part of treatment process of Claustrophobia by conducting of psychiatrist and psychologist experts.

In this regard, there are some challenges that should be considered. Providing a suitable computer system and VR glasses can be difficult because of high price in some countries. in addition, together with clinical experts, an 
effective therapy protocol should be prepared that determine the number of treatment sections and details of each section according to the patient's condition. Furthermore, it is necessary that the therapists have been trained in this field and acquired enough skill to use the software game corresponding to planned therapy protocol.

After clinical evaluation of the Claustrophobia Game, we hope this VR method can utilize in the treatment procedure of Claustrophobia patients.

\section{Financial support and sponsorship}

This research was supported by Isfahan University of Medical Sciences under grant number 195211.

\section{Conflicts of interest}

There are no conflicts of interest.

\section{References}

1. Davey GC. Phobias: A Handbook of Theory, Research, and Treatment. New York, USA: John Wiley \& Sons Inc.; 1997.

2. Abramowitz JS, Deacon BJ, Whiteside SP. Exposure Therapy for Anxiety: Principles and Practice.New York, USA: Guilford Press; 2012.

3. Bouchard S, Wiederhold BK, Loranger C. Claustrophobia: Efficacy and treatment protocols. In: Advances in Virtual Reality and Anxiety Disorders. Boston, MA: Springer US; 2014. p. $145-62$.

4. Ma M, Jain LC, Anderson P. Virtual, Augmented Reality and Serious Games for Healthcare 1. Vol. 68. Heidelberg, Germany, Springer; 2014.

5. Mandal S. Brief introduction of virtual reality \& its challenges. Int J Sci Eng Res 2013;4:304-9.

6. Halarnkar P, Shah S, Shah H, Shah H, Shah A. A review on virtual reality. Int J Comput Sci Issues 2012;9:325-30.

7. Fox J, Arena D, Bailenson JN. Virtual reality: A survival guide for the social scientist. J Med Psychol 2009;21:95-113.

8. North MM, North SM. Virtual reality therapy. In: Computer-Assisted and Web-Based Innovations in Psychology, Special Education, and Health. London: Academic Press; 2016. p. 141-56.

9. Ceranoglu TA. Video games in psychotherapy. Rev Gen Psychol 2010;14:141-6.

10. Bruce M, Regenbrecht H. A virtual reality claustrophobia therapy system - Implementation and test. In: Proceedings - IEEE Virtual Reality. Los Angeles, USA, IEEE; 2009. p. 179-82.

11. Malbos E, Mestre DR, Note ID, Gellato C. Virtual reality and claustrophobia: Multiple components therapy involving game editor virtual environments exposure. Cyberpsychol Behav 2008;11:695-7.

12. Shiban Y, Peperkorn H, Alpers GW, Pauli P, Mühlberger A. Influence of perceptual cues and conceptual information on the activation and reduction of claustrophobic fear. J Behav Ther Exp Psychiatry 2016;51:19-26.

13. Stănică IC, Dascălu MI, Moldoveanu A, Moldoveanu F. An innovative solution based on virtual reality to treat phobia. Int $\mathrm{J}$ Interact Worlds 2017;2017:1-13.

14. Botella C, Villa H, Baños R, Perpiná C, García-Palacios A. The treatment of claustrophobia with virtual reality: Changes in other phobic behaviors not specifically treated. Cyberpsychol Behav 1999;2:135-41.

15. Botella $\mathrm{C}$, Baños RM, Villa $\mathrm{H}$, Perpiñá C, García-Palacios A. Virtual reality in the treatment of claustrophobic fear: A controlled, multiple-baseline design. Behav Ther 2000;31:583-95.

16. Christofi M, Michael-Grigoriou D. Virtual environments design assessment for the treatment of claustrophobia. In: $201622^{\text {nd }}$ International Conference on Virtual System \& Multimedia (VSMM). Kuala Lumpur, Malaysia, IEEE; 2016. p. 1-8.

17. Spielberger CD, Gorsuch RL, Lushene PR, Vagg PR, Jacobs AG. Manual for the State-Trait Anxiety Inventory (Form Y). Palo Alto, CA: Consulting Psychologists Press; 1983.

18. Sánchez JL, Vela FL, Simarro FM, Padilla-Zea N. Playability: Analysing user experience in video games. Behav Inf Technol 2012;31:1033-54.

19. Sánchez JL, Zea NP, Gutiérrez FL. Playability: How to identify the player experience in a video game. In: Lecture Notes in Computer Science (Including Subseries Lecture Notes in Artificial Intelligence and Lecture Notes in Bioinformatics). LNCS, no. PART 1. Vol. 5726. INTERACT: IFIP Conference on HumanComputer Interaction, Uppsala, Sweden, IFIP; 2009. p. 356-9.

20. Nacke LE, Drachen A, Kuikkaniemi K, De Kort YA, Niesenhaus J, Korhonen HJ, et al. Playability and player experience research. In: Proceedings of DiGRA 2009: Breaking New Ground: Innovation in Games, Play, Practice and Theory. London, UK, DiGRA; 2009. p. 1-11.

21. Sánchez JL, Simarro FM, Zea NP, Vela FL. Playability as Extension of Quality in Use in Video Games. Proceedings of the Second International Workshop on the Interplay between Usability Evaluation and Software Development(I-USED'09) Uppsala, Sweden, IFIP; 2009. p. 1-6.

22. Nacke LE, Schild J, Niesenhaus J. Gameplay experience testing with playability and usability surveys - An experimental pilot study. In: Playability and player experience: Proceedings of the Fun and Games 2010 Workshop. Leuven, Belgium, NHTV Expertise Series 10, 2010. p. 31-45.

23. Baranowski T, Blumberg F, Buday R, DeSmet A, Fiellin LE, Green CS, et al. Games for health for children-current status and needed research. Games Health J 2016;5:1-2. 


\section{BIOGRAPHIES}

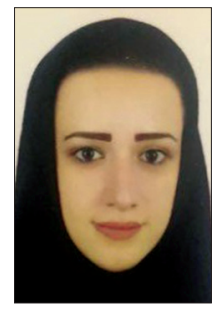

Vida Kabiri Rahani received her B.Sc. degree in information technology in 2013 from Salman Farsi University of Kazerun, Kazerun, Iran; and she obtained her master degree in information technology (IT) on e-commerce trend from Safahan Institute of Higher Education, Isfahan, Iran in 2016. Her research interests are design and development of serious games, virtual reality in medicine and medical image processing.

Email: amoabedini@safaiau.ac.ir

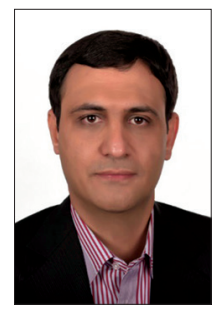

Alireza Vard received his B.Sc. degree in software engineering from University of Isfahan, Isfahan, Iran in 2004, and he got his M.Sc. and Ph.D. degrees in computer engineering from University of Isfahan, in 2007 and 2012 respectively. He is currently an assistant professor at Department of Biomedical Engineering, Isfahan University of Medical Sciences, Isfahan, Iran. His research interests include medical image processing, application of virtual and augmented reality in medicine, design and development of medical image processing software tools. Email:vard@amt.mui.ac.ir

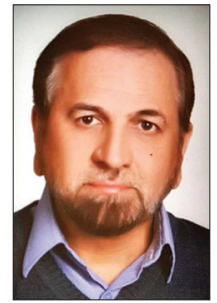

Mostafa Najafi received his MD degree and specialty in psychiatry from Isfahan University of Medical Sciences, Isfahan, Iran, in 1994 and 1997 respectively. He got his sub-specialty in childadolescence from Tehran University of Medical Science in 2005. He is now an associate professor at psychiatric department in Isfahan University of Medical Sciences. His research interests are in the fields of serious and therapeutic games, using technology and developing virtual reality and augmented reality in improving mental health.

Email: Najafimostafa@med.mui.ac.ir 